\title{
A importância do pré-natal na prevenção de complicações materno- fetais do diabetes mellitus gestacional
}

\author{
The importance of prenatal care in the prevention of maternal-fetal complications of \\ gestational diabetes mellitus
}

La importancia de la atención prenatal en la prevención de las complicaciones maternofetales de la diabetes mellitus gestacional

\begin{abstract}
Bruna da Silva Barros ${ }^{1 *}$, Bianca de Souza Nepomuceno², Lucas Barbosa Santana ${ }^{2}$, Maria Clara Leal Oliveira de Sá ${ }^{2}$, Maria Eduarda Venturim Almeida Vieira ${ }^{2}$, Mayra França Bendel ${ }^{3}$, Priscilla Pereira Prates Souza, Rafael Xavier Cunha ${ }^{5}$, Rodrigo Aparecido Guimarães ${ }^{2}$, Maria Luísa Barros Quintão Couto Parreira6 .
\end{abstract}

\section{RESUMO}

Objetivo: Revisar e demonstrar métodos de prevenção de complicações do Diabetes Mellitus Gestacional, por meio de intervenções e tratamentos para o controle glicêmico. Revisão bibliográfica: $O$ alto índice glicêmico durante o período gestacional poderá culminar em Diabetes Mellitus do tipo 2, o qual é originado por variados fatores, tais como mudanças hormonais, conjuntamente aos hábitos de vida da gestante. Tal patologia poderá gerar complicações para o feto se não diagnosticada e tratada precocemente. Destaca-se que o feto pode nascer com variadas complicações, sendo a mais frequente a macrossomia fetal, promovida pelo estímulo de uma maior produção e secreção de insulina, em decorrência do aporte elevado de glicose ao feto. Ademais, evidencia-se que a mãe poderá apresentar lesões durante o parto em consequência da macrossomia, além de um alto risco de desenvolvimento de Diabetes do tipo 2, doenças cardiovasculares, dentre outras. Sendo assim, nota-se a necessidade de um rastreamento das pacientes com diabetes gestacional, além de uma mudança no estilo de vida (MEV) da grávida. Considerações finais: Considera-se que o pré-natal é de suma importância para o rastreamento, diagnóstico e tratamento de pacientes com diabetes gestacional ou pré-disposição a patologia, evitando possíveis complicações.

Palavras-chave: Pré-natal, Diabetes gestacional, Relações materno-fetais.

\footnotetext{
ABSTRACT

Objective: Review and demonstrate methods for preventing complications of Gestational Diabetes Mellitus, through interventions and treatments for glycemic control. Bibliographic review: The high glycemic index during the gestational period may culminate in Type 2 Diabetes Mellitus, which is caused by several factors, such as hormonal changes, in conjunction with the pregnant woman's life habits. Such pathology can lead to complications for the fetus if not diagnosed and treated early. It is noteworthy that the fetus can be born with various complications, the most frequent being fetal macrosomia, promoted by stimulating a greater production and secretion of insulin, due to the high supply of glucose to the fetus. Furthermore, it is evident that the mother may present injuries during childbirth as a result of macrosomia, in addition to a high risk of developing type 2

1 Universidade do Grande Rio (UNIGRANRIO), Rio de Janeiro - RJ. *E-mail: Bruna.10barross@gmail.com

${ }^{2}$ Centro Universitário UNIFAMINAS, Muriaé - MG.

${ }^{3}$ Universidade Vila Velha (UVV), Vila Velha - ES.

${ }^{4}$ Faculdade de Minas (FAMINAS), Belo Horizonte - MG.

${ }^{5}$ Universidade Nilton Lins (UNL), Manaus - AM.

${ }^{6}$ Faculdade de Medicina do Vale do Aço (UNIVAÇO), Ipatinga - MG.
} 
diabetes, cardiovascular diseases, among others. Thus, there is a need for screening patients with gestational diabetes, in addition to a change in the pregnant woman's lifestyle (SEM). Final considerations: Prenatal care is considered of paramount importance for the screening, diagnosis and treatment of patients with gestational diabetes or pre-disposition to pathology, avoiding possible complications.

Key words: Prénatal, Gestational diabetes, Maternal-fetal relations.

\section{RESUMEN}

Objetivo: Revisar y demostrar métodos para prevenir complicaciones de la diabetes mellitus gestacional, mediante intervenciones y tratamientos para el control glucémico. Revisión bibliográfica: El alto índice glucémico durante el período gestacional puede culminar en Diabetes Mellitus tipo 2, que es causada por varios factores, como cambios hormonales, en conjunto con los hábitos de vida de la mujer embarazada. Dicha patología puede provocar complicaciones para el feto si no se diagnostica y trata a tiempo. Es de destacar que el feto puede nacer con diversas complicaciones, siendo la más frecuente la macrosomía fetal, promovida al estimular una mayor producción y secreción de insulina, debido al alto aporte de glucosa al feto. Además, es evidente que la madre puede presentar lesiones durante el parto como consecuencia de la macrosomía, además de un alto riesgo de desarrollar diabetes tipo 2, enfermedades cardiovasculares, entre otras. Por tanto, existe la necesidad de realizar un cribado de pacientes con diabetes gestacional, además de un cambio en el estilo de vida de la mujer embarazada (SEM). Consideraciones finales: La atención prenatal se considera de suma importancia para el cribado, diagnóstico y tratamiento de pacientes con diabetes gestacional o predisposición a patología, evitando posibles complicaciones.

Palabras clave: Prénatal, Diabetes gestacional, Relaciones materno-fetales.

\section{INTRODUÇÃO}

O Diabetes Mellitus Gestacional (DMG) é definido pela Associação Americana de Diabetes (ADA) como diabetes diagnosticada no segundo ou terceiro trimestre da gestação, não sendo evidente antes da gestação. O DMG é determinado como intolerância à glicose, tendo como consequência hiperglicemia de variável intensidade, com início ou primeiro diagnóstico durante a gravidez, podendo ou não persistir após o parto. $O$ diagnóstico do DMG ocorre habitualmente a partir de testes provocativos com cargas de glicose (NUNES RD, et al., 2019; TAVARES MGR, et al., 2019).

A Associação Internacional de Grupos de Estudo de Diabetes e Gravidez apresentou em 2010 um novo método de diagnóstico fundamentado no teste de tolerância oral à glicose de $75 \mathrm{~g}$, sendo realizado entre 24 e 28 semanas de gestação, com aferição da glicose plasmática no início em jejum, após 1 hora e após 2 horas e uma única medida alterada (glicose plasmática em jejum $\geq 92 \mathrm{mg} / \mathrm{dL} ; 1$ hora $\geq 180 \mathrm{mg} / \mathrm{dL} ; 2$ horas $\geq 153 \mathrm{mg} / \mathrm{dL}$ ) é suficiente para que seja realizado o diagnóstico de DMG (AMARAL AR, et al.,2015).

A prevalência geral de DMG é de 3-5\%, podendo atingir 18\%. A prevalência estimada no Brasil varia de $2,4 \%$ a $7,2 \%$. Nos Estados Unidos, entre as mulheres com baixo risco a doença atinge de $1,4 \%$ a $2,8 \%$ e entre as mulheres que apresentam algum fator de risco 3,3\% a 6,1\%. Segundo estudos abordando a evolução da prevalência de DMG nos últimos 10 anos, sugerem que essa doença terá aumento significativo. Além disso, cinco anos após a gravidez, pode ocorrer o desenvolvimento de diabetes mellitus tipo 2 entre essas mulheres diagnosticadas com DMG, levando em conta que é um fator predisponente para essa doença (TAVARES MGR, et al., 2019; NUNES RD, et al., 2019).

Diversas são as complicações que podem ocorrer advindas do DMG, pode haver trabalho de parto por um período prolongado, dificuldade do feto se adaptar a pelve da mãe, laceração de partes moles, aumento de cesarianas, hemorragias após o parto, atonia uterina levando a uma retenção placentária, fratura de clavícula do feto devido a macrossomia, comprometimento da inervação plexo braquial e decorrente disso pode gerar paralisia de Erb (TAVARES MGR, et al. 2019). 
É necessário que tenham medidas eficazes para controlar o ganho de peso na gestação, para prevenir danos neonatais e a obesidade infantil. Há algumas terapias utilizadas para obter melhorias no DMG e na obesidade, que são as mudanças nos hábitos de vida, através da adesão de dietas adequadas e realização de exercícios físicos. O sedentarismo e a alimentação descontrolada provocam um aumento e piora do DMG. (NASCIMENTO IB, et al., 2020; NIÑO GP, et al., 2019)

Além desses meios, há também as medidas farmacológicas, o cloridrato de metformina é um antidiabético oral que demonstra bons resultados na obesidade gestacional, o medicamento quando interligado as mudanças dos padrões alimentares e atividades físicas tem efeitos positivos, com melhora no nível glicêmico, pressóricos e redução de massa corpórea. O tratamento para DMG, tem como objetivo manter um controle glicêmico, para que assim tenha uma queda de morbidades e mortalidade da mãe e do feto, semelhante as gestações em que a mãe não possui DMG (NASCIMENTO IB, et al., 2020; NIÑO GP, et al., 2019).

O objetivo do presente estudo consiste em revisar e demonstrar os métodos de prevenções de complicações do DMG, por meio de intervenções e tratamentos para o controle glicêmico.

\section{REVISÃO BIBLIOGRÁFICA}

\section{Definição}

O DMG é proveniente da elevação do nível sérico de glicose, esta desordem metabólica é bastante comum no ciclo gravídico. Destaca-se que esse diagnóstico é dado quando a hiperglicemia é detectada pela primeira vez durante a gestação e tal patologia poderá ser fator de predisposição para o desenvolvimento de Diabetes Mellitus do tipo 2 (FEBRASGO, 2019).

A hiperglicemia observada no período gestacional poderá advir do processo de resistência insulínica em virtude das mudanças observadas no organismo, tais como o consumo de glicose pelo bebê e a presença de hormônios produzidos pela placenta. Além disso, hormônios como a prolactina e o cortisol, que estarão em taxas mais elevadas, poderão contribuir para uma resistência à insulina. Sendo assim, em condições fisiológicas normais a gestante irá produzir mais insulina como forma de suprir as demandas necessárias, no entanto, quando essa produção já está no limite, a elevação se torna insuficiente e aumentam as chances do surgimento de DMG (OPAS, et al., 2017).

Os hábitos de vida preliminares das gestantes têm papel preponderante no desenvolvimento do DMG. Sendo assim, pacientes obesas, principalmente acima de 25 anos, bem como com a presença de comorbidades como hipertensão, possuem maiores chances de desenvolvimento da patologia em questão e, desse modo, é possível graduar esse risco a partir de determinados critérios durante a triagem inicial, sendo observada a história familiar de Diabetes, seguida do IMC superior à $30 \mathrm{~kg} / \mathrm{m}^{2}$, além de parto pregresso de bebê com peso acima de $4 \mathrm{~kg}$, perda perinatal inexplicada e por fim, idade superior à 35 anos. Além disso, é notório o aumento do número de diagnósticos nos últimos anos que pode ser explicado pela piora dos hábitos alimentares atrelado à idade avançada das gestantes (NAKABUYE B, et al., 2017; BARROS, GM, et al., 2019).

Nesse sentido, evidencia-se a necessidade do diagnóstico precoce da DMG, bem como da adoção de medidas profiláticas que visem a diminuição da incidência desta patologia, tendo em vista que em 1 a cada 6 nascimentos é possível verificar a presença de distúrbios de hiperglicemia, sendo que em cerca de 5 dessas gestações os distúrbios seriam decorrentes do DMG (OPAS, et al., 2017; NETA, FAV, et al., 2019).

\section{Complicações para o bebê}

Inicialmente, é válido destacar que o DMG quando diagnosticado repercute até o fim da gravidez, principalmente quando o tratamento da patologia não é feito adequadamente. Nesse sentido, há risco de complicações para o bebê e até mesmo para a mãe que repercutem na saúde do feto, incluindo nascimento precoce, bem como a macrossomia fetal (peso superior a $4 \mathrm{~kg}$ ao nascer), pré-eclâmpsia, eclâmpsia, distocia de ombros durante o parto, dificuldade respiratória e até morte fetal. No entanto, tais repercussões fetais e neonatais podem ser evitadas caso seja realizado o diagnóstico precoce e o controle devido da patologia. $\mathrm{O}$ 
feto também poderá desenvolver malformações, icterícia e hipoglicemia neonatal. Ao tratar-se do risco de malformação fetal, conhecido como embriopatia, esta manifestação está diretamente relacionada ao nível de controle da glicose materna em fases muito iniciais da gestação, sendo os níveis de hemoglobina glicada (HbA1c) se associando direta e progressivamente ao risco de desenvolvimento. Portanto, recomenda-se que toda mulher com DMG seja alertada quanto à importância do acompanhamento e controle da doença a fim de evitar complicações (FEBRASGO, 2019; TIAN Y, et al., 2020).

Tendo em vista o aporte excessivo de glicose da mãe diabética para o feto, há um estímulo da produção de insulina fetal, culminando em crescimento fetal descontrolado. Dessa forma, os recém-nascidos, filhos de pacientes afligidos pela patologia, tendem a acumular gordura no organismo. Sendo assim, gestantes que apresentam DMG normalmente não são indicadas a passar pelo parto normal, sendo indicada a cesariana como forma de evitar o sofrimento intraparto causado pela macrossomia. Existem casos em que há a necessidade da internação em UTI neonatal, como em prematuridade, hipoglicemia neonatal, pré-eclâmpsia grave, trauma no parto, polidrâmnio e macrossomia. Evidencia-se ainda que o aumento da frequência a consultas para pré-natal reduz o número de nascimentos prematuros, e mesmo quando isso ocorre, a detecção precoce do DMG atrelada ao controle glicêmico aumenta a razão de chance de sobrevida dos recém-nascidos (SILVA R, et al., 2019).

\section{Complicações maternas}

Além das complicações na prole, a DMG pode ser responsável por diversas alterações maternas. O alto peso ao nascer está relacionado à maior taxa de lesões na mãe no ato do parto, como laceração perineal, laceração de bexiga, hemorragia pós-parto e retenções placentárias decorrentes da atonia uterina. Há também elevada ocorrência de infecções do trato urinário durante a gestação e predominância de partos cesáreos (BOZATSKI BL, et al., 2019; TAVARES MGR, et al., 2019; SAMPAIO Y, et al., 2021).

Mulheres com histórico de diabetes gestacional apresentam maior risco para desenvolver diabetes mellitus tipo 2. Esse grupo também é fator de risco para doenças cardiovasculares, como obesidade, hipertensão e dislipidemia. A doença hepática gordurosa não alcoólica, inflamação crônica, disfunção vascular e doença renal crônica são complicações que também podem ocorrer em pacientes portadoras de DMG (DENNISON RA, et al., 2020; SARAVANAN P, 2020).

A diabetes gestacional trata- se de uma preocupação de saúde global devido às possíveis implicações tanto materna como na sua prole. Dada então, a importância da realização do pré-natal, propiciando um acompanhamento saudável materno-fetal, prevenindo ou até mesmo tratando intercorrências que podem surgir durante a gestação (PEREIRA DO, et al., 2017; BOZATSKI BL, et al., 2019).

\section{Prevenção}

É essencial que a gestante esteja comprometida com o pré-natal, comparecendo às suas consultas com a periodicidade correta e realizando o controle glicêmico adequado ao momento gestacional para que o risco de desenvolvimento de DMG e suas complicações seja o menor possível. De acordo com o estudo de Nakabuye B, et al. (2017), foi identificado que a prevalência de hiperglicemia detectada pela primeira vez na gravidez foi de 31,9\%. Desse percentual, $95 \%$ das gestantes foram diagnosticadas com DMG e 5\% com DM 2. Existem medidas consideradas eficazes para a prevenção de DMG e suas complicações. Intervenções moderadas e individualizadas no estilo de vida são capazes de reduzir a incidência de DMG, especialmente em gestações de risco, influenciando diretamente na diminuição dos riscos de complicações tanto para a gestante quanto para o bebê (KOIVUSALO S, et al., 2017).

Dentre as mudanças no estilo de vida que podem prevenir a ocorrência de DMG existe a aplicação programada de exercícios físicos que contemplem treinos aeróbicos, exercícios que exigem mais coordenação, alongamentos e uso dos músculos do assoalho pélvico. Essas atividades devem ser iniciadas o quanto antes e mantidas durante a gestação, podendo reduzir o ganho excessivo de peso e o risco de DMG (BARAKAT R, et al., 2018).

Além disso, resultados ainda melhores são encontrados quando há a associação de alterações dietéticas com atividade física regular. A associação de uma dieta composta de frutas, vegetais, cereais integrais de 
acordo com a Nordic Nutrition Recommendations (NNR) como a dieta do mediterrâneo (MedDiet) com exercícios físicos regulares é considerada uma combinação influente na prevenção de DMG, ainda mais quando aplicada no período pré-gestacional e mantida durante a gravidez. Exercícios físicos aeróbicos e de resistência, praticados regularmente, possuem a capacidade de melhorar o controle glicêmico através do aumento da captação de glicose no músculo esquelético e da maior expressão mitocondrial de proteínas que transportam glicose (MIJATOVIC-VUKAS J, et al., 2018; KOKIC IS, et al., 2018).

\section{Tratamento}

Nos últimos anos, as estratégias preventivas e medicamentosas voltadas para o tratamento do DMG ganharam grande notoriedade, e sabendo que nas mulheres com diabetes pré-gestacional a melhor medida preventiva de complicações obstétricas e perinatais é o controle glicêmico rígido. Essa também será a base e o objetivo para as pacientes com o diagnóstico de DMG, pois as evidências sugerem que a intervenção nessas gestantes também reduz a ocorrência desses eventos adversos (HEDRINGTON M, et al., 2017).

\section{Medidas não farmacológicas}

Para prevenir ou fazer controle glicêmico adequado, as condutas inicialmente adotadas no DMG são medidas não farmacológicas. Dentre essas condutas, as mais importantes consistem no tratamento, prevenção e orientações quanto aos fatores de risco associados, como a idade materna, mães multíparas, mulheres com história familiar de diabetes ou de DMG, IMC pré-gestacional elevado, dentre outros. Além disso, cabe destacar que o controle metabólico e de ganho de peso gestacional deve ser acompanhado e controlado, por meio de orientações alimentares individualizadas e baseadas no cálculo calórico total da dieta, priorizando alimentos com carboidratos de baixo índice glicêmico, conduta que reduz a necessidade do uso de insulina no DMG (LIN P-C, et al., 2016).

Associado a dieta, a prática de exercícios físicos aeróbicos e/ou de força, acompanhado e assistido por profissional deve ser contemplado como parte do tratamento, pois as evidências sugerem que a associação das duas medidas possuem melhores resultados do que quando aplicado de forma individualizada, pois aumentam a sensibilidade esquelética e adiposa a insulina e ainda auxiliam na redução de peso. Por fim, deve-se buscar, tratar e controlar as comorbidades associadas, para que com isso a meta das medidas não farmacológicas sejam atendidas (LIN P-C, et al., 2016; SOCIEDADE BRASILEIRA DE DIABETES, 2020).

\section{Medidas farmacológicas}

As medidas farmacológicas são usadas em casos refratários às medidas anteriormente citadas, especificamente se após 2 semanas de dieta controlada os níveis glicêmicos permanecerem elevados. Sendo que, a metformina é uma opção a qual demonstra que os experimentos em animais não foram encontrados riscos, mas foram encontrados alguns efeitos colaterais não confirmados nas mulheres, especialmente durante o último trimestre, ou seja, recomendação de risco nível B na gestante, no entanto a primeira escolha permanece sendo a insulina (SBD, 2020).

\section{Metformina}

Atualmente é a única opção dentre os anti hiperglicemiantes orais usados na gestante, com recomendação categoria $B$, onde as evidências não demonstram efeitos deletérios materno-fetais e ainda exerce seus mecanismos sem fazer hipoglicemia ou ganho de peso, através da inibição de gliconeogênese hepática e aumento da sensibilidade à insulina. Apesar de existirem questionamentos quanto a possibilidade da sua passagem através da placenta e assim fazer ativação de genes associados ao diabetes tipo 2 na idade adulta, a Sociedade Brasileira de Diabetes aponta que não foram evidenciados eventos adversos graves a médio prazo, mas que ainda não existem estudos que demonstrem os reais efeitos metabólicos nesta população a longo prazo (SBD, 2020).

A relação metformina e insulina é destacada por diversos estudos, dentre eles, uma metanálise realizada por Kitwitee $\mathrm{P}$, et al. (2015), o qual demonstrou que o grupo com uso de metformina teve menor hipoglicemia neonatal, menor ganho de peso e menor necessidade UTI neonatal, quando comparado à insulina, mas que cerca de $14-46 \%$ destas, necessitaram de adição insulínica. 
Portanto, da comprovada eficácia insulínica e da sua pequena passagem placentária, a insulina ainda é a primeira escolha terapêutica para o DMG, estando a metformina reservada para casos especiais, como não acessibilidade a insulina, dificuldade na autoadministração e outras condições individualizadas (SOCIEDADE BRASILEIRA DE DIABETES, 2020; KITWITEE P, et al., 2015).

\section{Insulinoterapia}

A insulinoterapia é caracterizada como categoria B e permanece como primeira escolha no tratamento do DMG, sendo a dose inicial recomendada de $0,5 \mathrm{U} / \mathrm{Kg}$ com ajustes individualizados. Cabe destacar que alguns dos análogos da insulina comprovadamente possuem melhor perfil farmacocinético, farmacodinâmico, demonstram menor risco de hipoglicemia e melhor controle glicêmico (HEDRINGTON M, et al., 2017).

Dentre os análogos de ação rápida, os classificados como de categoria B são o LISPRO e ASPART. Estes demonstram vantagens sobre a insulina regular, comprovado por um estudo prospectivo randomizado realizado por Di Cianne G, et al. (2007), demonstrando que a insulina regular leva a maior chance de hipoglicemia e de alterações antropométricas neonatais. Já em relação aos análogos de insulina de ação prolongada, duas drogas são consideradas como opção, a Glargina, com recomendação C e o Detemir, pertencente à categoria $B$.

\section{CONSIDERAÇÕES FINAIS}

A partir da análise dos achados da literatura, verificou-se que o DMG ocasiona inúmeras consequências à saúde materno-fetal durante a gestação, parto e puerpério. A assistência pré-natal qualificada proporciona diagnóstico precoce e tratamento adequado, através de medidas farmacológicas e não farmacológicas, com consequente redução das complicações à saúde da mãe e do feto. Os cuidados devem ser constantes, desde o diagnóstico ao puerpério. Algumas medidas demonstraram-se eficazes para prevenção do DMG, devendo ser aconselhadas e incentivadas pelo médico assistente, sobretudo as pacientes com fatores de risco, com objetivo de reduzir a ocorrência dessa patologia de incidência crescente.

\section{REFERÊNCIAS}

1. AMARAL AR, et al. Impacto do diabetes gestacional nos desfechos neonatais: uma coorte retrospectiva. Scientia Medica, 2015; 25(1): 1-6.

2. BARAKAT R, et al. Exercise during pregnancy has a preventative effect on excessive maternal weight gain and gestational diabetes. A randomized controlled trial. Brazilian Journal of Physical Therapy, 2018;1: 1-8.

3. BARROS GM, et al. Idade como fator de risco para Diabetes Mellitus Gestacional, 2019; 3-7

4. BOZATSKI BL, et al. Epidemiological Profile of Diabetic Pregnancies at Itajaí City, SC. Arquivos Catarinenses de Medicina, 2019; 48(2): 34-55.

5. DENNISON RA, et al. The absolute and relative risk of type 2 diabetes after gestational diabetes: A systematic review and meta-analysis of 129 studies. Diabetes Research and Clinical Practice, 2020; 171: 108625-108637.

6. DI CIANNE G, et al. Maternal metabolic control and perinatal outcome in women with gestational diabetes mellitus treated with lispro or aspart insulin: comparison with regular insulin. Diabetes Care. 2007; 30(4): e11.

7. FEBRASGO. Diabetes Gestacional. Revista Feminina, 2019; 47(11).

8. HEDRINGTON MS, DAVIS SN. The Care of pregestational and gestational diabetes and drug metabolism considerations. Expert opinion on drug metabolism e toxicology, 2017; 3(10): 1029-1038.

9. KITWITEE P, et al. Metformin for the treatment of gestational diabetes: on update meta-analysis. Diabetes Res Clin Pract. 2015;109(3): 521-532.

10. KOIVUSALO S, et al. Gestational diabetes mellitus can be prevented by lifestyle intervention: the finnish gestational diabetes prevention study (RADIEL). Diabetes Care, 2015; 1: 1-7.

11. KOKIC IS, et al. Combination of a structured aerobic and resistance exercise improves glycaemic control in pregnant women diagnosed with gestational diabetes mellitus. A randomised controlled trial. Women and Birth, 2018; 31(4): e232-e238.

12. MIJATOVIC-VUKAS J, et al. Associations of Diet and Physical Activity with Risk for Gestational Diabetes Mellitus: A Systematic Review and Meta-Analysis. Nutrients, 2018; 1: 1-19.

13. NAKABUYE B, et al. Prevalence of hyperglycaemia first detected during pregnancy and subsequent obstetric outcomes at St. Francis Hospital Nsambya. BMC Research Notes, 2017; 10(174): 1-10.

14. NASCIMENTO IB, et al. Exercício físico e metformina na obesidade gestacional e prevenção diabetes mellitus gestacional: revisão sistemática. Revista Brasileira de Saúde Maternidade Infantil, 2020; 20(1): 17-26. 
15. NEHAB SRG, et al. Influence of gestational and perinatal factors on body composition of full-term newborns. Jornal de Pediatria, 2020; 96(6): 771-777.

16. NETA FAV, et al. Avaliação do perfil e dos cuidados no pré-natal de mulheres com diabetes mellitus gestacional. Revista Rene, 2014; 15(11).

17. NIÑO GP, et al. Intervenciones nutricionales para el tratamiento de la diabetes mellitus gestacional. Revista. Ciencia Salud, 2019; 17: 108-119.

18. NUNES RD, et al. Two criteria of oral glucose tolerance test to diagnose gestational diabetes mellitus. Revista Associação Médica Brasileira, 2020; 66(2): 139-145.

19. OPAS, et al. Rastreamento e diagnóstico de diabetes mellitus gestacional no Brasil, 2017; 7-28.

20. LIN P-C, et al. The risk factors for gestational diabetes mellitus: A retrospective study. Midwifery, 2016; $42: 16-20$.

21. PEREIRA DO, et al. Evaluation of prenatal consultations: prenatal access and complications in maternal-child health. Revista Ciência Plural, 2017; 3 (3): 2-15.

22. REICHELT AJ, et al . Clinical characteristics of women with gestational diabetes - comparison of two cohorts enrolled 20 years apart in southern Brazil. São Paulo Medical Journal, 2017; 135(4): 376-382.

23. SAMPAIO Y, et al. Gestational diabetes and overt diabetes first diagnosed in pregnancy: characteristics, therapeutic approach and perinatal outcomes in a public healthcare referral center in Brazil. Archives of Endocrinology and Metabolism, 2021; 65(1): 79-84.

24. SARAVANAN P. Gestational diabetes: opportunities for improving maternal and child health. Lancet Diabetes Endocrinol, 2020; 8: 793-800.

25. SOCIEDADE BRASILEIRA DE DIABETES. Diretrizes Sociedade Brasileira de Diabetes. Clannad, editora científica (online), 2020.

26. TAVARES MGR, et al. Profile of pregnant women with gestation diabetes mellitus at increased risk for large for gestational age newborns. Rev bras ginecol. obstet, 2019; 41: 298-305.

27. TIAN Y, et al. Glycemic qualification rate and frequency of self-monitoring blood glucose glycemic qualification rate and frequency of self-monitoring blood glucose (SMBG) in women with gestational diabetes mellitus (GDM). Diabetes Research and Clinical Practice, 2020; 170: 1-8. 